\title{
Can Confinement-Induced Variations in the Viscous Dissipation be Measured?
}

\author{
Sissi de Beer - Wouter K. den Otter • \\ Dirk van den Ende · Wim J. Briels • \\ Frieder Mugele
}

Received: 15 November 2011 / Accepted: 26 December 2011/Published online: 10 January 2012

(C) The Author(s) 2012. This article is published with open access at Springerlink.com

\begin{abstract}
Liquids confined to molecular scales become anisotropic and often show pronounced self-organization such as layering. Although this effect is well accepted, it is still debated if confinement induces measurable changes of viscous friction. We use molecular dynamics to address this issue by simulating a Lennard-Jones liquid confined between a solid cylinder and an atomically smooth surface. The simulations reproduce the well-established variations of normal force, density, and diffusivity with the distance between wall and cylinder. We find high diffusivity and low density when the numbers of layers is in between integers. This observation seems to contradict most experimental results on the effective damping between atomic force microscope tips and substrates when interpreting them within continuum hydrodynamics used to connect liquid viscosity and diffusivity. This contradiction is resolved by directly extracting the damping that the tip experiences, which we achieve by using the fluctuationdissipation theorem; as in experiment, we find local minima in the damping near integer numbers of molecular layers and maxima in between. These variations correlate with distinct structural changes in the microscopic order of the fluid. We reconfirm that constitutive equations valid at macroscopic scales cannot be used to interpret confined
\end{abstract}

\footnotetext{
S. de Beer $(\bowtie) \cdot$ D. van den Ende $\cdot$ F. Mugele Physics of Complex Fluids and MESA + Institute for Nanotechnology, Department of Science and Technology, University of Twente, P. O. Box 217, 7500 AE Enschede, The Netherlands

e-mail: s.j.a.debeer@utwente.nl

W. K. den Otter · W. J. Briels

Computational Biophysics, Department of Science and

Technology, University of Twente, P. O. Box 217,

7500 AE Enschede, The Netherlands
}

liquids and finally conclude that viscous friction displays measurable, non-monotonic behavior with the degree of confinement.

Keywords Nanotribology $\cdot$ Lubrication ·

Molecular dynamics

\section{Introduction}

The dissipation in liquid layers confined to a few nanometers plays a crucial role for friction experienced by lubricated contacts $[1,2]$. While there is overwhelming evidence that simple liquids can be described by standard continuum models using the bulk viscosity and density down to length scales of $10 \mathrm{~nm}$, deviations occur when the liquid thickness is reduced to a few molecular diameters [3]. Seminal work using the Surface Forces Apparatus (SFA) established that the presence of solid walls induces a layered structure in the liquid leading to the so-called oscillatory solvation forces [4]. X-ray reflectometry studies [5] as well as theory and numerical simulations [6, 7] demonstrated the existence of an oscillatory density profile, which decays approximately exponentially with a decay length of a few molecular diameters as a function of the distance from the wall. While this behavior of the conservative forces is well understood and has also been reproduced for nanoscale contacts in early Atomic Force Microscopy (AFM) experiments [8], the dissipative behavior of nano-confined liquid films is heavily debated. In the SFA community, slightly different measurement techniques for nominally identical systems produced highly incompatible experimental results with interpretations ranging from confinement-induced solidification [9] or a glassy response [10] to bulk-like dynamics for liquid 
films of even just a few molecular layers thickness [11-13]. SFA measurements are limited to film thicknesses of integer numbers of molecular layers due to a mechanical ("jump-to-contact") instability. In contrast, dynamic AFM allows for a more complete characterization of the conservative forces and the dissipation including in particular film thicknesses corresponding to non-integer numbers of molecular layers. Notwithstanding early controversies [1416], there is a growing consensus that the dissipation experienced in lubricated single-asperity nano-contacts varies in a non-monotonic manner as a function of the thickness of the confined liquid film: Qualitatively similar behavior was observed for a variety of liquids including the simple Lennard-Jones liquid Octamethylcyclotetrasiloxane (OMCTS) [17-19], dodecanol [20], and water [21] (also depending on the ion concentration [22]). Although, chemical details are known to qualitatively alter the friction forces [23, 24], this similarity in observations using a large variety of liquids with rather disparate molecular properties and solid-liquid interactions [25] suggests the presence of a generic underlying principle.

In this letter, we describe equilibrium molecular dynamics (MD) simulations of a confined Lennard-Jones liquid that mimics the experimental conditions of AFM experiments with OMCTS and graphite surfaces. Early non-equilibrium simulations [26, 27], applied to study the dissipative properties, were often performed at high shear rates while only for sufficiently low velocities the experimentally examined Stokes friction can be extracted [28]. Instead, we analyze the temporal fluctuations of the force and make use of the fluctuation-dissipation theorem to extract the dissipation from our equilibrium simulations. Therefore, and in contrast to previous equilibrium MD simulations of confined liquids [29, 30], our study is not limited to the equilibrium properties such as density profiles, oscillatory solvation forces, and diffusivities. In this manner, we reproduce for the first time the distance dependence of the damping measured in AFM experiments.

\section{Background and Technical Aspects}

Figure 1 shows the typical experimental situation and experimental result that we want to study in our simulations. Such AFM experiments are carried out in dynamic mode where the AFM cantilever is driven to oscillate sinusoidally at $\mathrm{kHz}$ frequencies in the vertical direction with an amplitude that is small compared to the diameter of the molecules. The resulting motion of the AFM cantilever is affected by both the conservative and the dissipative forces mediated by the confined liquid underneath the tip. The average distance between tip and sample is slowly decreased at a typical speed of $<1 \mathrm{~nm} / \mathrm{s}$. Measuring the inphase and the out-of-phase component of the response of the AFM cantilever, the conservative interaction stiffness $k_{\text {int }}$ and the dissipative interaction damping $\gamma_{\text {int }}$ can be extracted (see e.g. [18]). As shown in Fig. 1c, $\gamma_{\text {int displays }}$ pronounced maxima at the distances of the minima of the stiffness. Despite the dynamic character of the experiments, it should be noted that the typical time scales for generating an experimental data point $(\sim 10 \mathrm{~ms})$ are very long compared to molecular time scales. As a consequence it is plausible to assume that the experimental data correspond to a succession of equilibrium states.

To mimic this situation numerically, we built the system sketched in Fig. 2. It consists of two solid surfaces of graphite, each consisting of at least ten layers of atoms, all fixed to their lattice sites in the characteristic honeycomb structure of individual graphene sheets. The AFM tip is mimicked by a shell of LJ atoms, equally fixed to their fcc

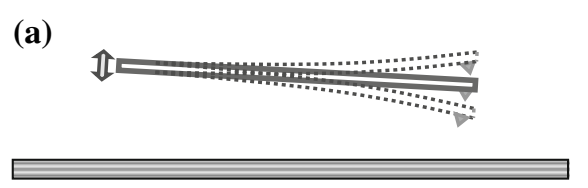

(b)

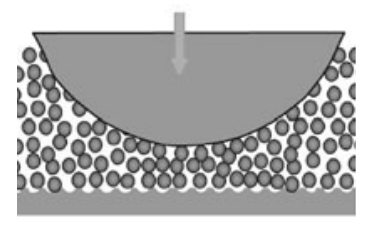

Fig. 1 a Oscillating AFM cantilever. b The liquid confined between the tip and the surface. c Measured interaction stiffness $k_{\text {int }}$ and damping $\gamma_{\text {int }}$ extracted from the amplitude phase distance curves

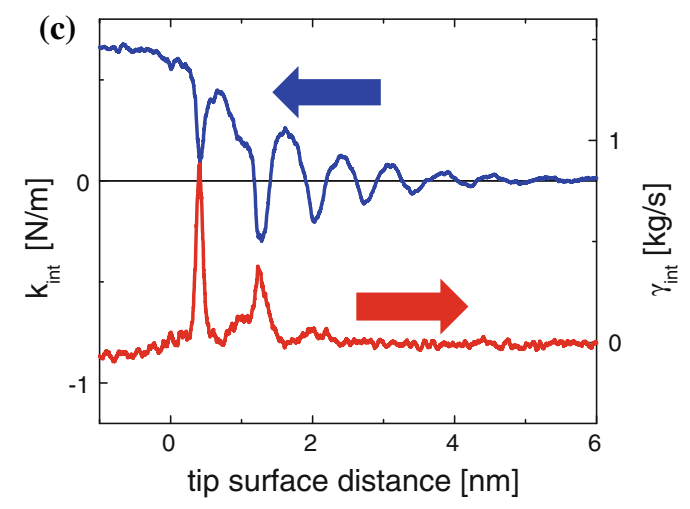

measured upon approach of the AFM cantilever towards HOPG in OMCTS (same data as in Ref. [18]) 


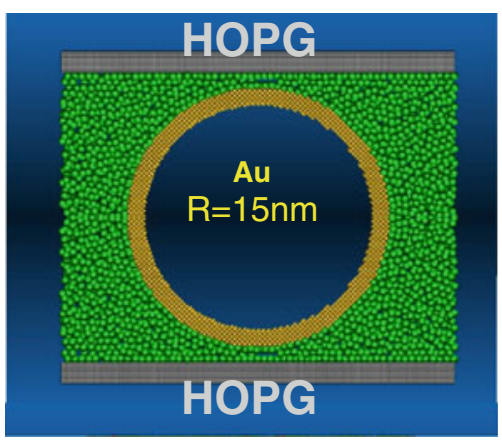

Fig. 2 Setup for the MD simulations. The green LJ particles in the liquid region represent OMCTS, yellow particles in the solid shell represent gold, and the gray particles represent graphite. Note the two confinement volumes at the top and the bottom of the box. The confined liquid is in equilibrium with the bulk liquid visible at the left and right side of the box (Color figure online)

lattice sites. This fec crystal is cut into various shapes including a cylinder (radius: $15 \mathrm{~nm}$ ) with a flat (100) bottom face, with curved bottom faces having the radii of 15 and $45 \mathrm{~nm}$, and into a sphere. The liquid molecules are represented by Lennard-Jones particles. All particles in the system interact via a Lennard-Jones potential $V(r)=4 \varepsilon_{\mathrm{i}}\left(\left(\sigma_{\mathrm{i}} / r\right)^{12}-\left(\sigma_{\mathrm{i}} / r\right)^{6}\right)$, where $\varepsilon_{\mathrm{i}}$ and $\sigma_{\mathrm{i}}$ are the interaction strength and particle sizes for particles $i$ (tip: $\sigma_{\mathrm{t}}=0.3 \mathrm{~nm}, \varepsilon_{\mathrm{t}}=6.84 \mathrm{~kJ} / \mathrm{mol}$, substrate: $\sigma_{\mathrm{s}}=0.142 \mathrm{~nm}$, $\varepsilon_{\mathrm{s}}=0.3598 \mathrm{~kJ} / \mathrm{mol}$ and liquid: $\sigma_{1}=0.77 \mathrm{~nm}, \varepsilon_{1}=2.85$ $\mathrm{kJ} / \mathrm{mol}[31]$ ) in the system. Commensurability will significantly affect the frictional forces [26, 32, 33]. Thus, we tested and found that our solid walls and the liquid are all incommensurate. For the interactions between different kinds of particles we used the Lorentz-Berthelot combining rules and the interactions are cut-off at a distance of 2.5 molecular diameters.

The tip is placed in the middle of the simulation box creating two gaps of equal width at the top and at the bottom of the graphite surface, respectively. While the tip is cylindrically symmetric, the simulation box is cubic (lateral dimensions $\sim 45 \times 45 \mathrm{~nm}$ ) with periodic boundary conditions in $x$ and $y$. The simulations are carried out as follows: the system containing approximately 100.000 liquid molecules is first equilibrated for $60 \mathrm{~ns}$ at a constant temperature and pressure of $T=0.88 \varepsilon / k_{\mathrm{B}}$ and $P=3.3$ $\varepsilon / \sigma^{3}$, respectively. Since confinement can move the liquidsolid transition line in the phase diagram [34], the vicinity to the melting point may affect our results, in the simulations and experiments alike. Temperature and pressure are kept constant using Toxvaerds implementation of the Nosé-Hoover thermostat and barostat [35]. Integration of the equations of motion is carried out using the leap-frog Verlet algorithm [36]. Following equilibration, barostat and thermostat are turned off and we let the system evolve at constant particle number $N$, volume $V$, and energy $E$ for another $16 \mathrm{~ns}$. The forces acting on the tip are calculated from these runs at constant $N, V, E$. Simulations are carried out for various spacings between the solid surfaces leading to gap widths $D$ ranging from 0.5 to $3.9 \mathrm{~nm}$.

\section{Results}

We first analyze the equilibrium properties of the system. Figure 3a shows the density profiles of the liquid averaged over the two gaps for a cylindrical tip with a flat bottom/top at a variety of gap widths. The liquid displays strong density oscillations as expected. Note that there is a slight asymmetry between the density maxima adjacent to the graphite surface and the tip surface, which arises from the different strength of the interaction parameters. The average force exerted on the tip by the liquid molecules displays the expected oscillatory shape and decays to zero at the largest distances (see green squares in Fig. 3b). The orange triangles in Fig. $3 \mathrm{~b}$ show the number of atoms in the gap. Note that this curve displays pronounced plateaus with a fixed number of molecules over a substantial range of gap widths. This behavior reflects the stability of the molecular layers. As the gap width is varied, these layers remain intact without changing their internal structure (density in two dimensions) substantially. Merely, the average distance between the layers is decreased (see also Fig. 3a). During this compression the average density in the gap increases by $>10 \%$. Only upon exceeding a certain maximum density, retaining a fixed layer structure becomes unfavorable and the system adopts a new configuration with one molecular layer less. As shown in Fig. 3b, the plateau regions with a well-defined number of molecular layers and the transition regions with a more disordered structure are approximately equally wide for this configuration of a cylindrical tip.

For spherical tips, plateau and transition regions become much more blurred (data not shown). Nevertheless, snapshots of the density of the molecules confined between a spherical tip $\left(R_{\text {tip }}=15 \mathrm{~nm}\right)$ and a flat surface, time-averaged over the entire simulation run, still display a clear layer structures (Fig. 4b-f). For gap width of 4.3, 4.0, and $3.6 \sigma$ four layers are clearly discernible under the tip. At the largest gap width the molecules in the middle appear as a diffuse band. This reflects their high mobility on the time scale of the simulations. At the lower gap widths, the atoms become more localized and appear as bright spots, like the atoms in the layers immediately adjacent to the solid surfaces. Since the atoms do not move during the entire simulation run, the system may be considered as crystallized under these conditions. 
Fig. 3 a Equilibrium film density profiles for different tip surface distances (indicated in $\mathrm{nm}$ by the numbers on the left). b The total average force on the tip (green) squares and the total number of particles confined between the tip and the surface (orange) triangles (same data as in Ref. [41]) (Color figure online)

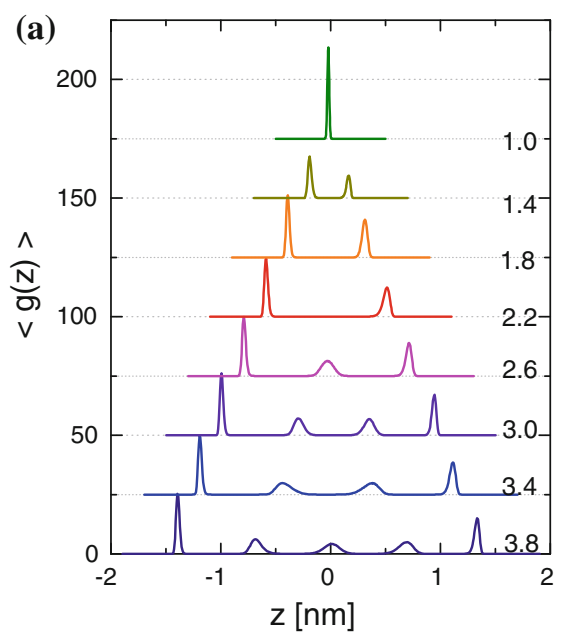

(b)

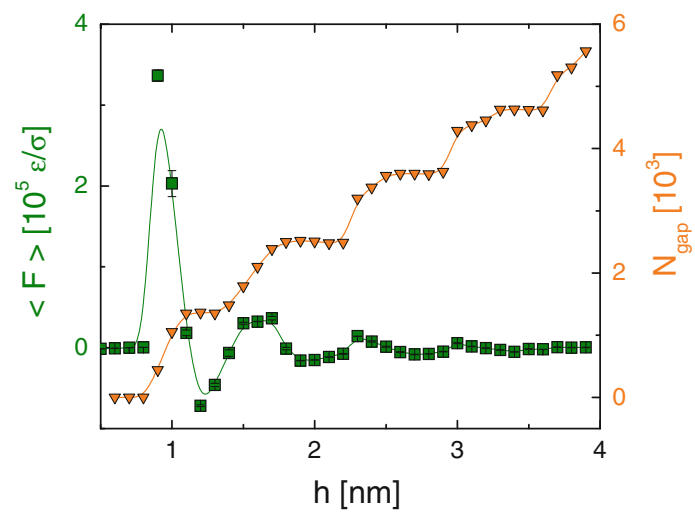

To quantify the variations of the atomic mobility we trace the mean square displacements of the atoms in the region underneath the spherical tip. This analysis reveals two aspects: first, the mean square displacements (MSDs) increase linearly in time within the time window of the simulations, both for the $x-y$ and for the $z$-direction. The linear behavior of the MSDs allows for the definition of diffusion coefficients $D_{\mathrm{xy}}$ and $D_{\mathrm{z}}$, being the slope of the means square displacement versus time. Second, the MSDs are highly anisotropic (see Fig. 4a). While $D_{\mathrm{xy}}$ and $D_{\mathrm{z}}$ are small compared to the bulk diffusion coefficient, $D_{\mathrm{xy}}$ is still substantially larger than $D_{\mathrm{z}}$. Qualitatively, a reduction of the diffusivity is of course expected. Already macroscopic fluid dynamics predicts a reduction of the diffusivity due to the interaction of the diffusing atom with its image on the opposite side of the wall [37-39]. Yet, the variations observed here are non-monotonic and reflect the layered structure of the liquid. The increase in density under the tip-and hence the decrease in space available for the siteexchange processes required for molecular diffusion-lead to a dramatic reduction of the diffusivity as the layers are compressed. Once the gap width is reduced to such an extent that the number of layers is reduced, the average density decreases again and molecules can diffuse faster again (Fig. 4e).

While the variations of the diffusivity are substantial, they are most pronounced at rather large separations, where the dissipation measurements do not reveal any structure yet (cf. Fig. 1c). Moreover, we observe an increased diffusivity for distances with a low density. Using continuum theory to directly convert the diffusivity to a viscosity, would imply an increased damping for distances with a high density. While in AFM experiments the opposite is often observed. Furthermore, it is well known that tip and liquid diffusivities do not have to agree [40]. Thus, a comparison of AFM data and MD simulations cannot be done on the level of diffusivities [41]. A comparison is only directly possible on the level of the damping that the AFM tip is expected to experience as a consequence of the molecular motion under the tip. To this end, we note that the forces exerted by the liquid onto the cantilever are not constant but fluctuate in time. On picosecond time scales these fluctuations are not completely random but display clear correlations: if some fraction of the molecules collectively moves in one direction at time $t_{0}$ and thereby exerts, e.g., an excess force, $\Delta F\left(t_{0}\right)=F\left(t_{0}\right)-\langle F\rangle$ then its excess force also points in the same direction at time $t_{0}+\Delta t$, provided that $\Delta t$ is sufficiently short compared to the relaxation time of the system. This memory of the fluid determines the macroscopic dissipation in the system at long times. So using the fluctuation-dissipation theorem [42], the macroscopic damping coefficient $\gamma$ on the long experimental time scales is given by the time integral of the autocorrelation function of the force fluctuations [43-45]:

$\gamma=\lim _{t \rightarrow \infty} \frac{1}{k_{\mathrm{B}} T} \int_{0}^{\mathrm{t}}\langle\Delta F(0) \Delta F(t)\rangle \mathrm{d} t$

It turns out that the force fluctuations display a strong autocorrelation for gap widths that correspond to the transition between two adjacent plateaus of particle numbers [41]. At these distances, the correlations display a rather long life time of several ns. In contrast, in the plateau regions, the correlation is rather weak and decays quickly on the ps time scale. Figure 5a shows the resulting damping coefficient for a flat cylindrical tip as a function of thickness for the motion in the vertical direction $\left(\gamma_{z}-\right.$ blue squares) and for the lateral motion $\left(\gamma_{x y}\right.$-red triangles). For gap widths below $2.5 \mathrm{~nm}$ pronounced variations as a function of the thickness are found for $\gamma_{\mathrm{z}}$. In the plateau regions of Fig. 3b, the damping coefficient of Fig. 5a is small and similar to the value at large distances. In the transition regions; however, $\gamma_{\mathrm{z}}$ is enhanced by one, two, and three orders of magnitude for $3 \leftrightarrow 2,2 \leftrightarrow 1$, and 


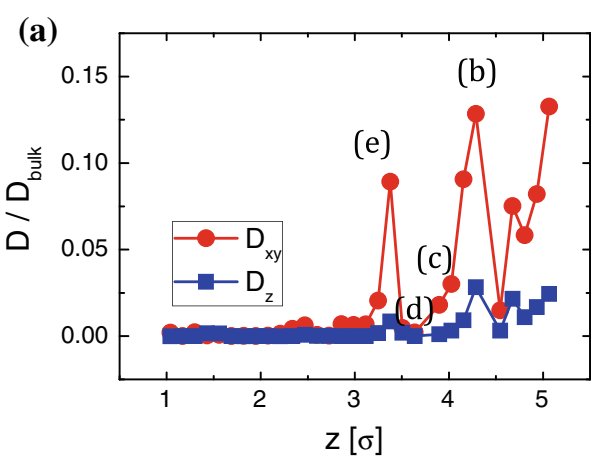

(b)

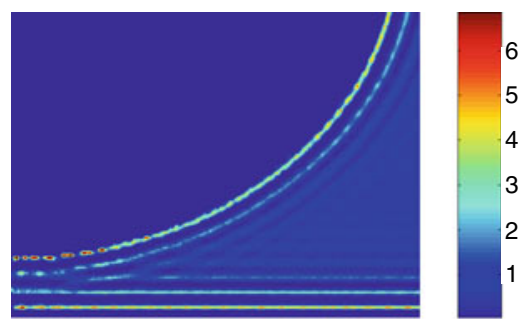

(c)

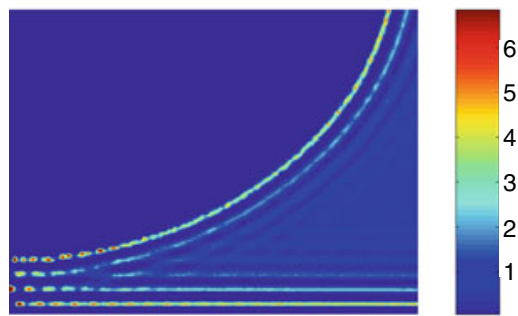

(d)

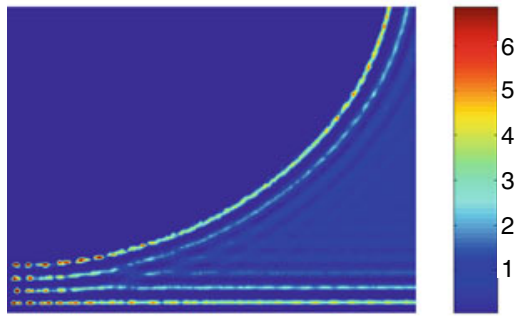

(e)

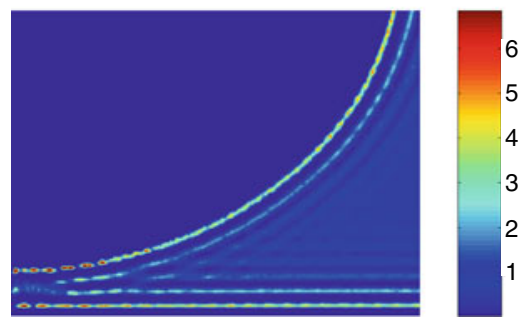

Fig. 4 Diffusion coefficient (blue) squares: $z$-direction; (red) circles: $x y$-direction) (a) and the normalized density (b-e) for various tip surface distances: $\mathbf{b} z=4.3 \sigma, \mathbf{c} 4.0 \sigma, \mathbf{d} 3.6 \sigma$ and $\mathbf{e} 3.4 \sigma$ (Spherical tip with bottom radius of curvature of $15 \mathrm{~nm}$ ) (Color figure online)

$1 \leftrightarrow 0$ layers, respectively. Simultaneously, the relaxation time of the system for motion along the $z$-direction varies between at most $100 \mathrm{ps}$ in the plateau regions and 1-2 ns in the transition regions (Fig. 5b). In contrast, the dissipation coefficient for the lateral dynamics, $\gamma_{\mathrm{xy}}$,
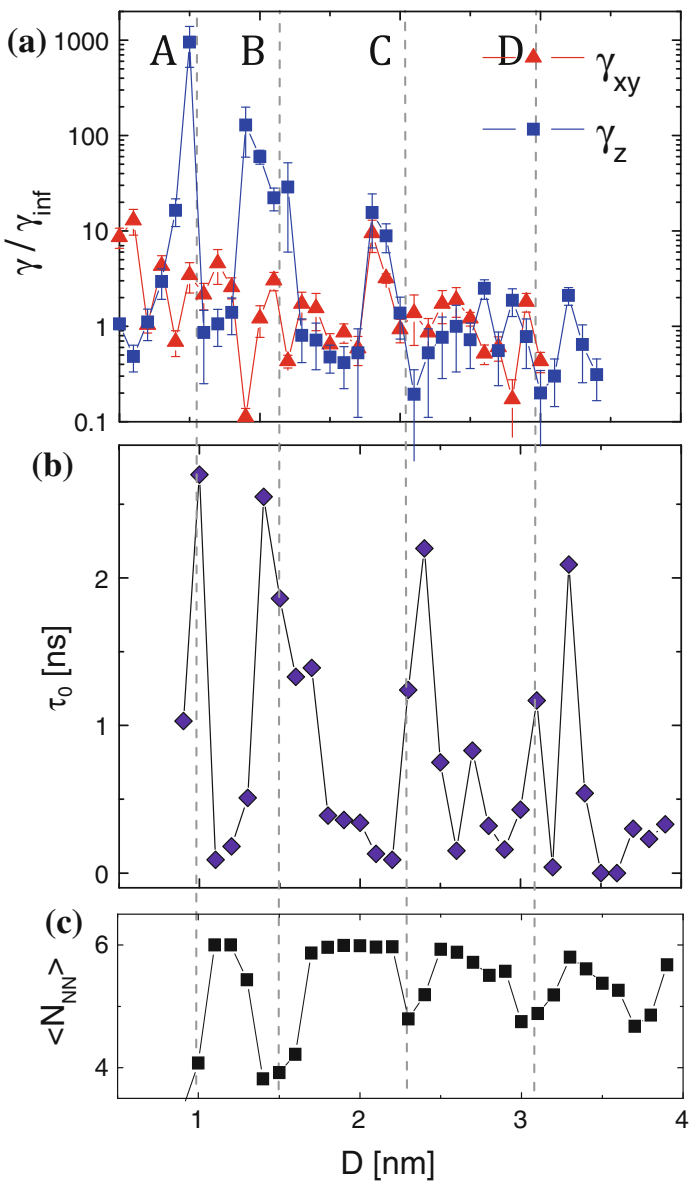

Fig. 5 a The damping coefficient $\gamma$ (red) triangles in the vertical $z$-direction and the inplane $x y$-direction ((blue) squares) both normalized by the average damping in between the peaks $\gamma_{\text {ref }}$ (same data as in Ref. [41]). $\mathbf{b}$ The characteristic relaxation time $\tau_{0}$. $\mathbf{c}$ The in plane number of nearest neighbors $\left\langle N_{\mathrm{NN}}\right\rangle$ averaged over all the particles under the tip. Gray dashed lines indicate the positions of the maxima of the repulsive force coinciding with maxima in the damping. All data were extracted from simulations with a cylindrical tip with a flat bottom surface (Color figure online)

displays within the noise no observable enhancement in the transition regions $2 \leftrightarrow 1$ and $1 \leftrightarrow 0$. Note that this result is consistent with the maximum of tenfold increase observed for non-commensurate systems in non-equilibrium simulations [46].

These variations in the damping and relaxation time are strongly correlated with accompanying variations in the structure of the film. Figure $5 \mathrm{c}$ shows that the number of nearest neighbors in the film varies strongly as a function of the thickness. In the plateau regions, the films are highly ordered with a sixfold coordination. In contrast, in the transition regions the films are more disordered and the number of nearest neighbors within the layer decreases down to four. Snapshots of the atoms in the layers reflect that picture (Figs. 6, 7). In particular, Fig. 6 illustrates rather the complex patterns arising when two hexagonal 

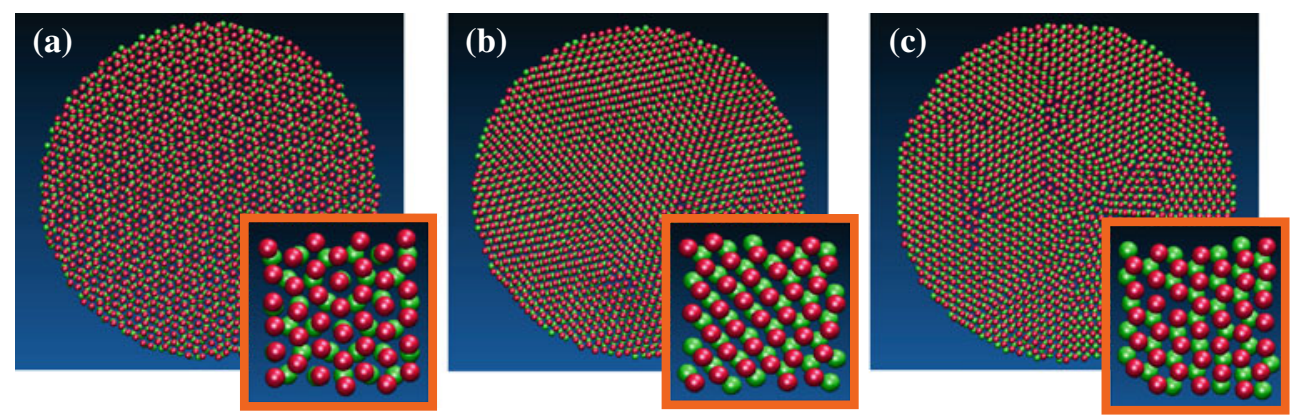

Fig. 6 Configuration of the molecules confined between the cylindrical tip with a flat bottom/top and the surface for 2 molecular layers $(r<15 \mathrm{~nm})$ at $\mathbf{a} D=2.2 \mathrm{~nm}, \mathbf{b} 1.9 \mathrm{~nm}$ and $\mathbf{c} 1.7 \mathrm{~nm}$. The insets show an amplification of the central region under the tip $(|x| \&|y|<3.85 \mathrm{~nm})$
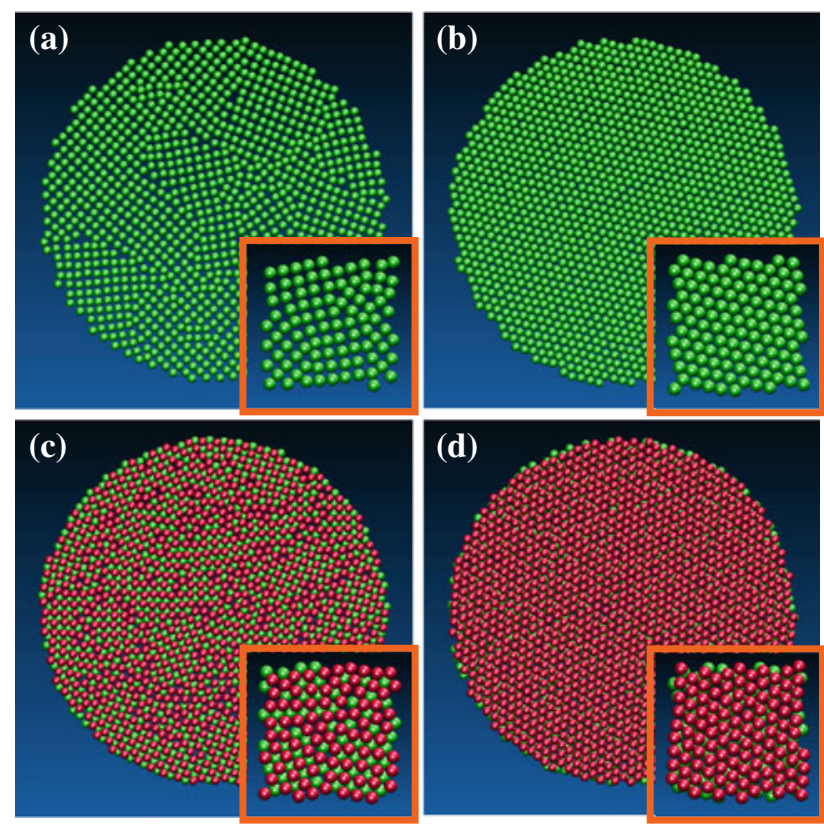

Fig. 7 Snapshots of the molecules confined between the cylindrical tip with a flat bottom/top and the surface $(r<15 \mathrm{~nm})$. a configuration at $D=1.0 \mathrm{~nm}, \mathbf{b} 1.1 \mathrm{~nm}, \mathbf{c} 1.5 \mathrm{~nm}$ and $\mathbf{d} 2.0 \mathrm{~nm}$. The insets show an amplification of the central region under the tip $(|x| \&|y|<3.85 \mathrm{~nm})$

layers are stacked on top of each other at different degrees of confinement: Fig. 6a shows the configuration of molecules at $D=2.2 \mathrm{~nm}$. There are two layers between the tip and the surface. Each layer has an in-plane hexagonal structure. Since $\sigma_{1}=0.77 \mathrm{~nm}$, there is a lot of free space in the gap. At this distance the hexagonal lattices of the two layers are free to move with respect to each other. When the distance is decreased to $D=1.9 \mathrm{~nm}$ (Fig. 6b), $N_{\text {gap }}$ remains the same and the molecules are in the same inplane hexagonal structure. However, the orientation of the two layers with respect to each other has changed. The molecules in the top layer mostly reside in between two molecules in the lower layer. In some regions, the molecules in the top layer occupy the threefold hollow sites as in the regular stacking of close-packed crystals. When the distance is decreased further to $D=1.7 \mathrm{~nm}$ (Fig. 6c), $N_{\text {gap }}$ is still the same and the molecules are still overall in the same in-plane hexagonal structure. However, almost all molecules in the top layer now reside in the threefold hollowsites of the bottom layer. Yet, the in-plane hexagonal structure is no longer perfect, but shows defects and grain boundaries and in some regions the molecules have positioned themselves in an in-plane quadratic arrangement with only 4 nearest neighbors. This tendency is enhanced when $D$ is further reduced and the transition to just one layer is approached. These transitions in the layer structure are reminiscent of the structural variations in confined liquid films between elastic substrates reported earlier by Persson [33, 47].

\section{Discussion}

The results presented here provide the first numerical confirmation of the rather generic experimental observation that the dissipation in confined liquid films shows distinct maxima and minima as a function of the gap width. Moreover, the maxima in the damping are found at transition distances $3 \leftrightarrow 2,2 \leftrightarrow 1$ and $1 \leftrightarrow 0$, very similar as observed experimentally. Throughout all our simulations, we found no indication that the system can get arrested in non-equilibrium states. The system always converged towards the structures shown above, irrespective of the initial conditions. From a physical perspective, this may not seem surprising given the typical time scales of molecular motion and the small size of the system. Yet, various experimental studies in the literature reported a strong dependence of the mechanical response of the liquid under investigation on the (very slow) approach rate of the cantilever $(\sim \AA / s)[17,21]$. A qualitatively similar dependence was reported in SFA experiments [10]. We suspect that this dependence is related to the rather large lateral dimensions of the confinement region in these experiments: The tips in the two AFM experiments were rather blunt with tip radii of order $>500 \mathrm{~nm}$ [48]. Under such 
conditions, it is easier to conceive that the molecules get "jammed" into some non-equilibrium configuration than for the system studied here, which displays nano-scale dimensions in all directions.

It is also interesting to compare the present results to previous interpretations of so-called confinement-induced solidification. For the present system, the material in the gap clearly crystallizes provided that the gap width corresponds to one of the plateau regions. At the same time, the dissipation due to the confined film remains at a very low level-and is in fact compatible with the same (noise) level that is also found at the largest gap widths: The dissipation of a thin solid film is just as low as for a thicker liquid film of small viscosity. This indicates that the dissipation experienced by an AFM cantilever oscillating in the vertical direction is not sensitive to a possible solidification of the layer. Similar conclusions apply for the tangential $x y$ motion. Distinguishing between the two states seems to require a different experimental technique.

The data shown in Fig. 5 refer to a cylindrical tip with a flat bottom. Unless a specific treatment is given to the tips (see e.g. [49]), this deviates from the typical experimental situation in AFM with round tips. Quite generally, one expects that the effects described above become smeared out when the cylindrical tip is replaced by a spherical one, because the net force experienced by the tip results from an average of regions with varying gap width. Figure 8 shows that this is indeed the case for two examples, namely cylinders for which the bottom sides were rounded with radii of curvature of $45 \mathrm{~nm}$ (a) and $15 \mathrm{~nm}$ (b), respectively. Despite that the data is still preliminary, it is clear that both the amplitude of the oscillatory solvation forces as well as the local maxima in $\gamma$ are less pronounced (note the different scale on the ordinate as compared to Fig. 5a). In fact, the decrease of the oscillatory forces between Fig. 8a and b is roughly one-third and consistent with a constant value of $F / R$ ( $R$ being the tip radius), as expected based on the Derjaguin approximation [50]. For the sharp tip of $15 \mathrm{~nm}$ radius, most of the variations in the damping have disappeared. Only one pronounced peak is left, which is related to the squeeze-out of the last layer. The latter, however, might be somewhat artificial in our simulations since the substrates in our model are perfectly stiff. In reality, the finite elasticity of primarily the soft graphite surface is likely to affect the squeeze-out process [33, 47]. Moreover, the specific shape of the tip will affect the pressure distribution in the contact area and friction forces [51,52] and is thus expected to affect the structure and dissipation in our system as well. Experiments suggest that the relative position of the peaks in the damping with respect to the conservative forces depends on the tip geometry [19]. In the simulations the shift was less pronounced. This might
Fig. 8 The average force on the tip in z-direction (blue) squares and the damping $\gamma$ extracted from the force fluctuations for a tip with radius a $R_{\text {tip }}=45 \mathrm{~nm}$ and $\mathbf{b} R_{\text {tip }}=15 \mathrm{~nm}$ (Color figure online)
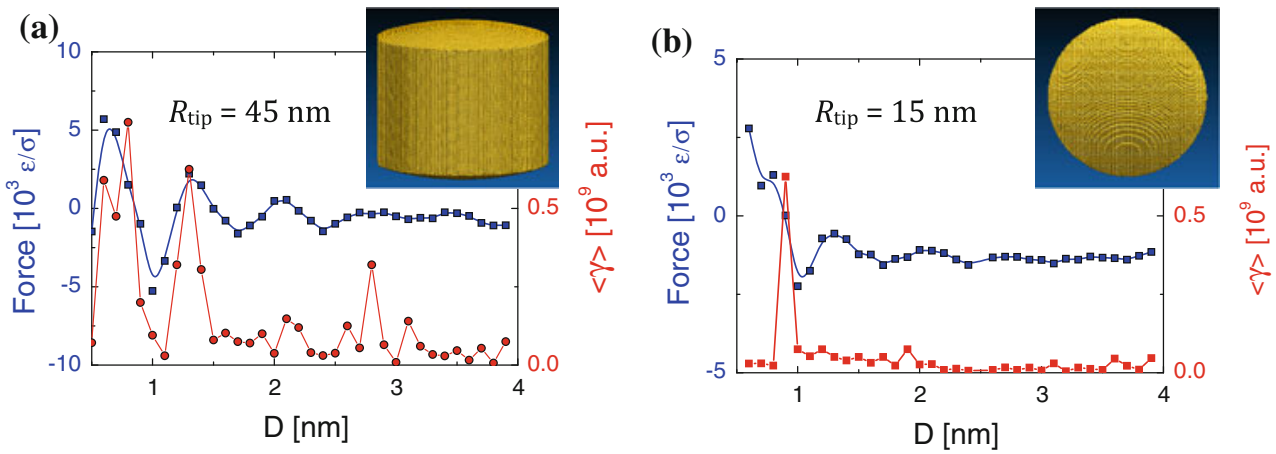

Fig. 9 The storage $C^{\prime}$ and loss $\mathrm{C}^{\prime \prime}$ moduli extracted from the autocorrelation of the force on the cylindrical tip with a flat bottom/top surface for a $D=1.5$ and $\mathbf{b} 1.9 \mathrm{~nm}$
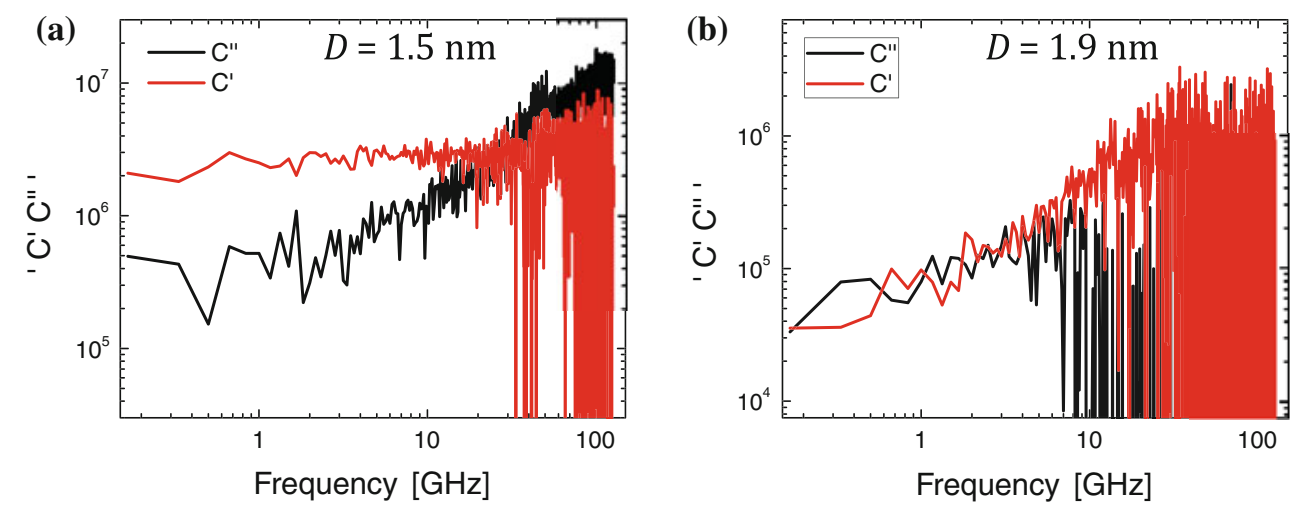
be related to the more complex geometry of real tips. This again implies, that the exact tip shape will have a significant effect on the measured damping.

Finally, we would like to comment on an alternative way of characterizing the "rheological" properties of the confined films, as it is frequently studied in experiments as well $[10,53]$. By calculating the Fourier transform of the force-autocorrelation function, we can extract the conservative and the dissipative response of the system for the entire spectrum of frequencies. In a macroscopic system, this procedure yields the frequency-dependent storage and loss modulus, $G^{\prime}$ and $G^{\prime \prime}$, respectively, of a system. The corresponding quantities $\mathrm{C}^{\prime}$ and $\mathrm{C}^{\prime \prime}$ for the present system are shown in Fig. 9a for $D=1.5 \mathrm{~nm}$ (i.e. in the transition region $2 \leftrightarrow 1$ ) and in Fig. 9 b for $D=1.9 \mathrm{~nm}$ (i.e. in the 2 layer plateau region). These results are again characteristic for other data obtained in transition and in plateau regions, respectively. Clearly, the mechanical response at both distances quite different. The storage modulus $\mathrm{C}^{\prime}$ in Fig. 9a, which represents the elastic response of the confined liquid molecules on the tip, is approximately constant over this frequency range. On the other hand, the loss modulus $\mathrm{C}^{\prime \prime}$, which represents the dissipative properties measured by the tip, increases linearly with slope 1 . This response is very close to the typical characteristics a soft glassy material [54]. This conclusion is consistent with the exponential, Maxwellian decay found for the autocorrelation in the force fluctuations (data not shown), which indicates liquid-like behavior for low frequencies. Although, longer simulations are required to confirm this. Figure $9 \mathrm{~b}$ shows $\mathrm{C}^{\prime}$ and $\mathrm{C}^{\prime \prime}$ for $D=1.9 \mathrm{~nm}$ (in between the peaks in the damping). Both the storage modulus and the loss modulus increase with a slope of approximately 0.5 . This response indicates gel-like behavior and the existence of several relaxation processes. The linear increase of both $\mathrm{C}^{\prime}$ and $\mathrm{C}^{\prime \prime}$ is consistent with the algebraic decay we found in the autocorrelation of the force fluctuations (data not shown).

These results indicate the behavior of the liquid is more complex than just a viscous liquid or an elastic solid. However, further research and especially longer simulations are required.

\section{Summary}

In summary, we showed that MD simulations of simple confined Lennard-Jones fluids are able to reproduce the characteristic peaks of excess dissipation that were found in a series of recent AFM experiments on confined fluids. The simulations suggest a generic relation between the dissipation measured in AFM experiments and the molecular scale packing of the molecules in the confined liquid layers. Highly ordered layers display little dissipation-a less ordered structure entails excess dissipation.

Acknowledgments The authors thank M. H. Müser for the discussions and his help on the manuscript. This work has been supported by the Foundation for Fundamental Research of Matter (FOM), which is financially supported by the Netherlands Organization for Scientific Research (NWO).

Open Access This article is distributed under the terms of the Creative Commons Attribution Noncommercial License which permits any noncommercial use, distribution, and reproduction in any medium, provided the original author(s) and source are credited.

\section{References}

1. Persson, B.N.J., Mugele, F.: Squeeze-out and wear: fundamental principles and applications. J. Phys. Condens. Matter 16, R295 (2004)

2. Müser, M.H., Urbakh, M., Robbins, M.O.: Statistical mechanics of static and low-velocity kinetic friction. Adv. Chem. Phys. 126, 187 (2003)

3. Chan, D.Y.C., Horn, R.G.: The drainage of thin liquid films between solid surfaces. J. Chem. Phys. 83, 5311 (1985)

4. Horn, R.G., Israelachvili, J.N.: Direct measurement of forces due to solvent structure. Chem. Phys. Lett. 71, 192 (1980)

5. Mo, H., Evmenenko, G., Dutta, P.: Ordering of liquid squalane near a solid surface. Chem. Phys. Lett. 415, 106 (2005)

6. Henderson, D., Abraham, F.F., Barker, J.A.: The Ornstein-Zernike equation for a fluid in contact with a surface. Mol. Phys. 31, 1291 (1976)

7. Snook, I.K., van Megen, W.: Structure of dense liquids at solid interfaces. J. Chem. Phys. 70, 3099 (1979)

8. O'Shea, S.J., Welland, M.E., Pethica, J.B.: AFM of local compliance at the liquid-solid interface. Chem. Phys. Lett. 223, 336 (1994)

9. Klein, J., Kumacheva, E.: Confinement-induced phase-transitions in simple liquids. Science 269, 816 (1995)

10. Zhu, Y., Granick, S.: Reassessment of solidification in confined fluids between mica sheets. Langmuir 19, 8148 (2003)

11. Becker, T., Mugele, F.: Nanofluidics: viscous dissipation in layered liquid films. Phys. Rev. Lett. 91, 166104 (2003)

12. Bureau, L., Arvengas, A.: Drainage of a nanoconfined simple fluid: rate effects on squeeze-out dynamics. Phys. Rev. E 78, 061501 (2008)

13. Bureau, L.: Nonlinear rheology of a nanoconfined simple fluid. Phys. Rev. Lett. 104, 218302 (2010)

14. Maali, A., Cohen-Bouhacina, T., Couturier, G., Aimé, J.-P.: Oscillatory dissipation of a simple confined liquid. Phys. Rev. Lett. 96, 086105 (2006)

15. O'Shea, S.J.: Comment on: oscillatory dissipation of a simple confined liquid. Phys. Rev. Lett. 97, 179601 (2006)

16. Maali, A., Cohen-Bouhacina, T.: Maali and Cohen-Bouhacina reply-comment on: oscillatory dissipation of a simple confined liquid. Phys. Rev. Lett. 97, 179602 (2006)

17. Patil, S., Matei, G., Oral, A., Hoffmann, P.M.: Solid or liquid? Solidification of a nanoconfined liquid under nonequilibrium conditions. Langmuir 22, 6485 (2006)

18. de Beer, S., van den Ende, D., Mugele, F.: Dissipation and oscillatory solvation forces in confined liquids studied by smallamplitude atomic force spectroscopy. Nanotechnology 21 , 325703 (2010) 
19. de Beer, S., van den Ende, D., Mugele, F.: Confinement-dependent damping in a layered liquid. J. Phys. Condens. Matter 23, 112206 (2011)

20. Hofbauer, W., Ho, R.J., Hairulnizam, R., Gosvami, N.N., O’Shea, S.J.: Crystalline structure and squeeze-out dissipation of liquid solvation layers observed by small-amplitude dynamic AFM. Phys. Rev. B 80, 134104 (2009)

21. Khan, S.H., Matei, G., Patil, S., Hoffmann, P.M.: Dynamic solidification in nanoconfined water films. Phys. Rev. Lett. 105, 106101 (2010)

22. Ulcinas, A., Valdre, G., Snitka, V., Miles, M.J., Claesson, P.M., Antognozzi, M.: Shear response of nanoconfined water on muscovite mica: role of cations. Langmuir 27, 10351 (2011)

23. Mugele, F., Persson, B.N.J., Zilberman, S., Nitzan, A., Salmeron, M.: Frictional properties of chain alcohols and the dynamics of layering transitions. Tribol. Lett. 12, 123 (2002)

24. Kong, L.T., Denniston, C., Müser, M.H.: The crucial role of chemical detail for slip-boundary conditions: molecular dynamics simulations of linear oligomers between sliding aluminum surfaces. Model. Simul. Mater. Sci. Eng. 18, 034004 (2010)

25. de Beer, S., Wennink, P., van der Weide-Grevelink, M., Mugele, F.: Do epitaxy and temperature affect oscillatory solvation forces? Langmuir 26, 13245 (2010)

26. Thompson, P.A., Robbins, M.O.: Origin of stick-slip motion in boundary lubrication. Science 250, 792 (1990)

27. Diestler, D.J., Schoen, M., Cushman, J.H.: On thermodynamic stability of confined thin films under shear. Science 262, 545 (1993)

28. Müser, M.H.: Nature of mechanical instabilities and their effect on kinetic friction. Phys. Rev. Lett. 89, 224301 (2002)

29. Gao, J., Luedtke, W.D., Landman, U.: Layering transitions and dynamics of confined liquid films. Phys. Rev. Lett. 79, 705 (1997)

30. Mittal, J., Truskett, T.M., Errington, J.R., Hummer, G.: Layering and position-dependent diffusive dynamics of confined fluids. Phys. Rev. Lett. 100, 145901 (2008)

31. Somers, S.A., McCormick, A.V., Davis, H.T.: Superselectivity and solvation forces of a two component fluid adsorbed in slit micropores. J. Chem. Phys. 99, 9890 (1993)

32. He, G., Müser, M.H., Robbins, M.O.: Adsorbed layers and the origin of static friction. Science 284, 1650 (1999)

33. Persson, B.N.J., Ballone, P.: Squeezing lubrication films: layering transition for curved solid surfaces with long-range elasticity. J. Chem. Phys. 112, 9524 (2000)

34. Kaneko, T., Mima, T., Yasuoka, K.: Phase diagram of LennardJones fluid confined in slit pores. Chem. Phys. Lett. 490, 165 (2010)

35. Toxvaerd, S.: Molecular dynamics at constant temperature and pressure. Phys. Rev. E 47, 343 (1993)
36. Frenkel, D., Smit, B.: Understanding molecular simulation. From algorithms to applications. Academic Press, San Diego (1996)

37. Brenner, H.: The slow motion of a sphere through a viscous fluid towards a plane surface. Chem. Eng. Sci. 16, 242 (1961)

38. Eral, H.B., Oh, J.M., van den Ende, D., Mugele, F., Duits, M.H.G.: Anisotropic and hindered diffusion of colloidal particles in a closed cylinder. Langmuir 26, 16722 (2010)

39. Imperio, A., Padding, J.T., Briels, W.J.: Diffusion of spherical particles in microcavities. J. Chem. Phys. 134, 154904 (2011)

40. Müser, M.H., Robbins, M.O.: Conditions for static friction between flat crystalline surfaces. Phys. Rev. B 61, 2335 (2000)

41. de Beer, S., den Otter, W.K., van den Ende, D., Briels, W.J., Mugele, F.: EPL, submitted

42. Chandler, D.: Introduction to modern statistical mechanics. Oxford University Press, Oxford (1987)

43. Bocquet, L., Barrat, J.-L.: Hydrodynamic boundary conditions, correlation functions, and Kubo relations for confined fluids. Phys. Rev. E 49, 3079 (1994)

44. Falk, K., Sedlmeier, F., Joly, L., Netz, R.R., Bocquet, L.: Molecular origin of fast water transport in carbon nanotube membranes: superlubricity versus curvature dependent friction. Nano Lett. 10, 4067 (2010)

45. Delhommelle, J., Cummings, P.T.: Simulation of friction in nanoconfined fluids for an arbitrarily low shear rate. Phys. Rev. B 72, 172201 (2005)

46. Müser, M.H.: Theory and simulation of friction and lubrication. Lect. Notes Phys. 704, 65 (2006)

47. Tartaglino, U., Sivebaek, I.M., Persson, B.N.J., Tosatti, E.: Impact of molecular structure on the lubricant squeeze-out between curved surfaces with long range elasticity. J. Chem. Phys. 125, 014704 (2006)

48. Hoffmann, P.M.: Private communications

49. Ebeling, D., van den Ende, D., Mugele, F.: Electrostatic interaction forces in aqueous salt solutions of variable concentration and valency. Nanotechnology 22, 305706 (2011)

50. Horn, R.G., Israelachvili, J.N.: Direct measurement of structural forces between two surfaces in a nonpolar liquid. J. Chem. Phys. 75, 1400 (1981)

51. Luan, B.Q., Robbins, M.O.: The breakdown of continuum models for mechanical contacts. Nature 435, 929 (2005)

52. Mo, Y.F., Turner, K.T., Szlufarska, I.: Friction laws at the nanoscale. Nature 457, 1116 (2009)

53. Li, T.-D., Riedo, E.: Nonlinear viscoelastic dynamics of nanoconfined wetting liquids. Phys. Rev. Lett. 100, 106102 (2008)

54. Purnomo, E.H., van den Ende, D., Vanapalli, S.A., Mugele, F.: Glass transition and aging in dense suspensions of thermosensitive microgel particles. Phys. Rev. Lett. 101, 238301 (2008) 